\title{
Management elements for the spatial organization of enterprises from the metallic materials industry
}

\author{
Mihnea Costoiu ${ }^{1}$, Adrian Ioana ${ }^{1, *}$, Augustin Semenescu ${ }^{1}$, Dragos Marcu ${ }^{1}$ and Massimo \\ Polifroni ${ }^{2}$ \\ ${ }^{1}$ University Politehnica of Bucharest, Spl. Independentei 313, Bucharest, Romania \\ ${ }^{2}$ University of Turin, Via Verdi, 8 - 10124 Turin, Italy
}

\begin{abstract}
The spatial organization of enterprises is of great importance for the metallic materials industry (metallurgy), given the diversity and complexity of the technological processes and the related equipment. In an enterprise from the metallic materials industry, there may be sections for: production, casting, forging, mechanical (hot and / or cold) processing, assembly etc. for the different stages of the technological process related to the products to be made. The article presents theoretical and practical elements regarding the management of the spatial organization of enterprises in the metallic materials industry. The principles and methods of spatial organization are presented and analyzed. These principles and methods are based on the biunivocal relation between the functional and constructive changes related to the technologies and equipment (machinery, aggregates) specific to the metallic material industry. Prescribing (establishing) the objective function (OF) of the technological processes modeling system in the metallic materials industry is based on the qualitative - economic analysis of these processes.
\end{abstract}

\section{Introduction}

The spatial organization of enterprises is of great importance for the metallic material industry (metallurgy), given the diversity and complexity of the technological processes and related machinery [1-3].

In an enterprise of the metallic materials industry, we can have sections for: elaboration, casting, forging, mechanical processing (hot and / or cold), assembly etc. for the various stages of the technological process related to the products to be made.

Each production unit will in turn have workshops in which machinery of the type appropriate to the respective technological process will operate. Thus, in an electric steelworks, for example, we can organize sectors (workshops) for: preparation - dosing of load, elaboration, casting, stripping, thermal treatment, expedition. Stripping is the technological process of hot extracting of steel ingots.

\footnotetext{
*Corresponding author : adyioana@gmail.com
} 
The main advantages of applying the principles and methods of spatial organization are the following $[4,5]$ :

$>$ Creating the optimal conditions for the rhythm of the production process.

$>$ Ensuring of higher flexibility in the use of the production means.

$>$ In case of machinery failure, the production process will not be disturbed.

$>$ Ensuring an efficient management of the production unit.

\section{Principles and methods of spatial organization}

The principles and methods of spatial organization of enterprises in the materials industry have to be based on the biunivocal relationship between the functional and constructive changes related to the specific technologies and equipment (machinery, aggregates), [3]. This biunivocal relationship is shown schematically in Figure 1.

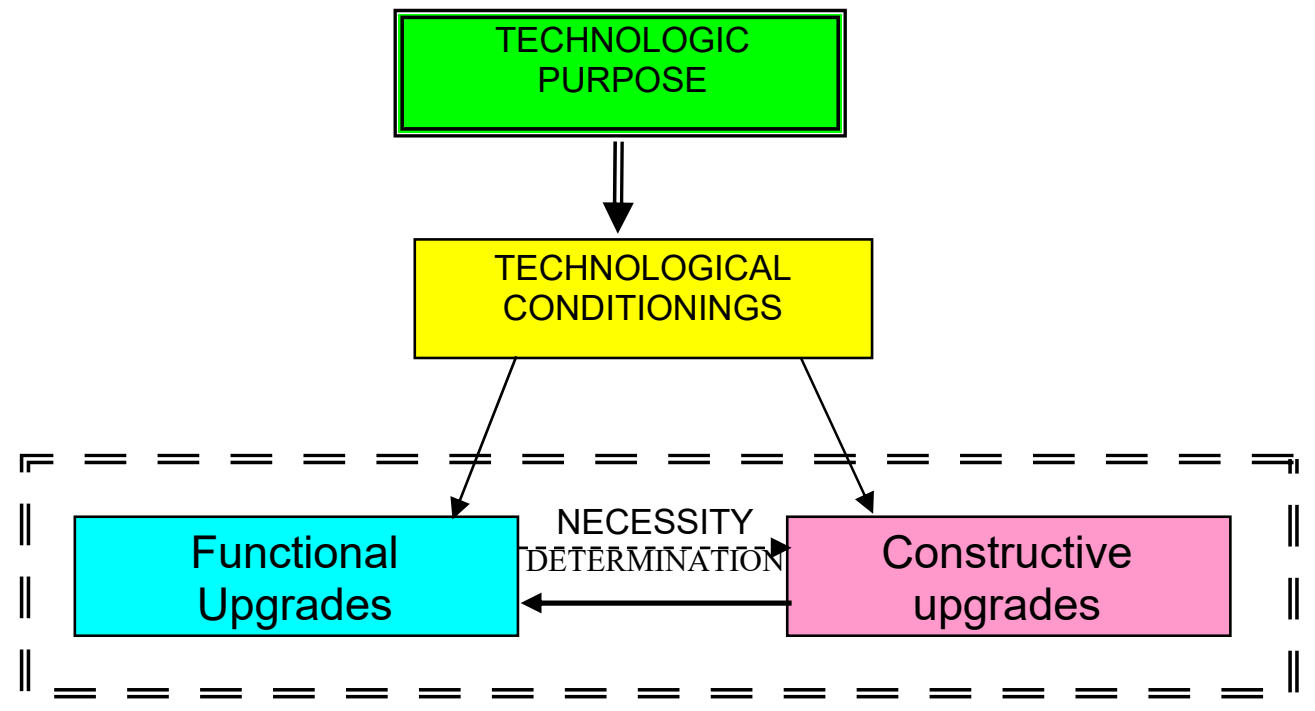

Fig. 1. The biunivocal relationship functional upgrade - constructive upgrade (technologically conditioned).

Of the principles and methods of spatial organization management we remark:

The technological purpose is given primordial importance.

Technological prescriptions (conditionings) are subordinated to the achievement of the proposed technological goal.

Technological prescriptions are obtained (achieved) by conception and implementation of the related functional and constructive upgrades.

Functional upgrades require specific constructive upgrades.

Constructive upgrades determine functional upgrades

A successful management of the space organization of the enterprises in the metallic materials industry requires research and design, including the use of mathematical modeling tools, by applying the following principles:

A. The principle of analogy - involves the observation and competent analysis of the modeled reality, using both the analogy with other fields of research and the logical homology.

According to this principle, to develop the mathematical models we have to go through these stages: 
Definition of the modeled object - this represents the primordial stage of the modeling analysis; this stage must satisfy both the purpose and the objectives of the system simultaneously with ensuring their compatibility.

$>$ Definition of efficiency criteria - it is a stage conditioned by the correct definition of the system objectives and allows the optimization of the modeling solutions.

$>$ Elaboration of options - based on the access to realistic, efficient and original solutions.

$>$ Evaluation of variants - according to established efficiency criteria.

$>$ Establishing of the final solution - based on the comparative analysis of the different solutions to which modeling leads.

B. The principle of concepts - based on the concepts of the systems theory including the concept of feed-back.

C. The principle of hierarchy - involves the necessity of building a hierarchical models system in order to structure the decision and to coordinate the interactive subsystems.

D. The principle of uncertainty - it is generated mainly by the high degree of complexity of the technological processes in the metallic materials industry. In addition, the existence of the interaction between the component subsystems - an interaction that cannot always be exactly predetermined - and the nonlinearities existing in the system, the subjectivity of the choice and the prescription of the objective function contribute to the increase of the uncertainty factors.

It is useful to note that based on this modeling principle, as the complexity of the system increase, requiring a hierarchical structure, the developed models decrease in precision but increase in the degree of relevance.

E. The principle of the internal model - establishes that a dynamic system is structurally stable only if:

$>$ It uses the negative reaction of adjusted quantities.

$>$ It incorporates in the reaction loop a reduplicated model of the dynamic structure of the exogenous signals; this internal model provides the signals designed to asymptotically compensate for the perturbations of the analyzed technological system.

The prescription (the establishing) of the objective function (OF) for the technological processes modeling system in the metallic materials industry is based on the qualitative economic analysis of these processes.

In this respect, the development of a new product (in this case a quality steel brand) has to ensure both its profitability and its ability to provide quality.

The conception of the mathematical model for prescribing the objective function is based on the quantification of the objective function (OF) in the form of a qualitativeeconomic matrix $\mathrm{M}_{\mathrm{QE}}$, according to the scheme shown in Figure 2.

The objective function prescribing levels are obtained by applying a three-vector composition algorithm:

- Vector $\overline{\mathrm{T}}$ - technical parameters vector $\left(\mathrm{t}_{\mathrm{i}}\right)$.

- Vector $\bar{E}$ - economic parameters vector $\left(\mathrm{e}_{\mathrm{j}}\right)$.

- Vector $\overline{\mathrm{P}}$ - weightings vector $\left(\mathrm{p}_{\mathrm{k}}\right)$. 


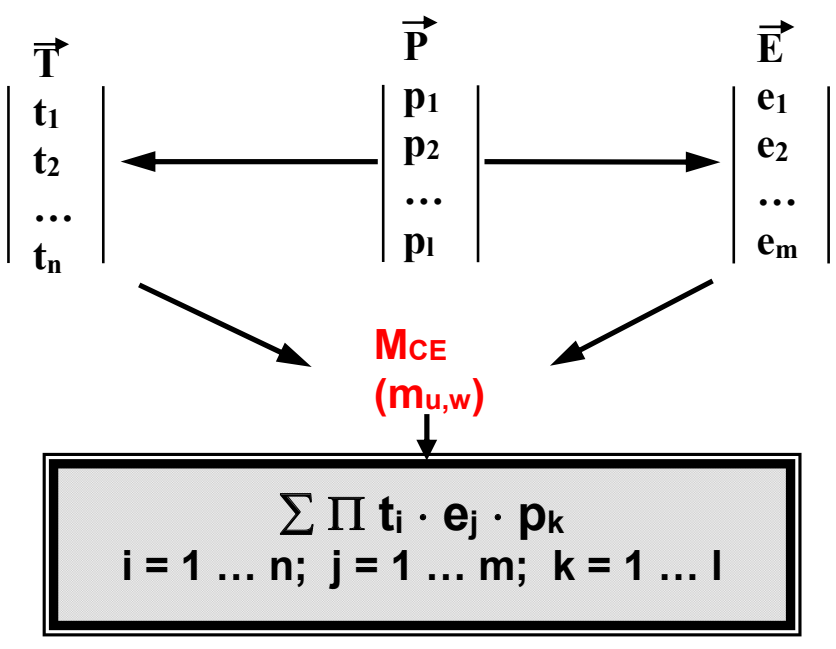

Fig. 2. Quantification of the modeling system objective function.

The components of the two vectors $\overline{\mathrm{T}}$ and $\overline{\mathrm{E}}$ considered with important weightings in the quantification (prescription) of the objective function OF are adopted directly depending on the fixed technological purpose (from which the necessary technological conditionings result).

The following is an example of adopting the components of the two vectors in the case of the technological process for the electric steel elaboration.

The components of the technical parameters vector $\left(t_{i}\right)$ :

$>\mathrm{t}_{1}$ - the chemical composition of the steel (marking precision);

$>t_{2}$ - the purity of the steel (in gases);

$>t_{3}$ - the purity of the steel (in inclusions).

The components of the economic parameters vector $\left(\mathrm{e}_{\mathrm{j}}\right)$ :

$>\mathrm{e}_{1}$ - the specific consumption of raw materials and materials;

$>\mathrm{e}_{2}$ - the specific energy consumption;

$>\mathrm{e}_{3}$ - the productivity of the elaboration process in the EAF.

\section{The spatial organization of an electric steelworks}

A relevant example of spatial organization for the specific case of an electric steelworks is shown in Figure 3. 


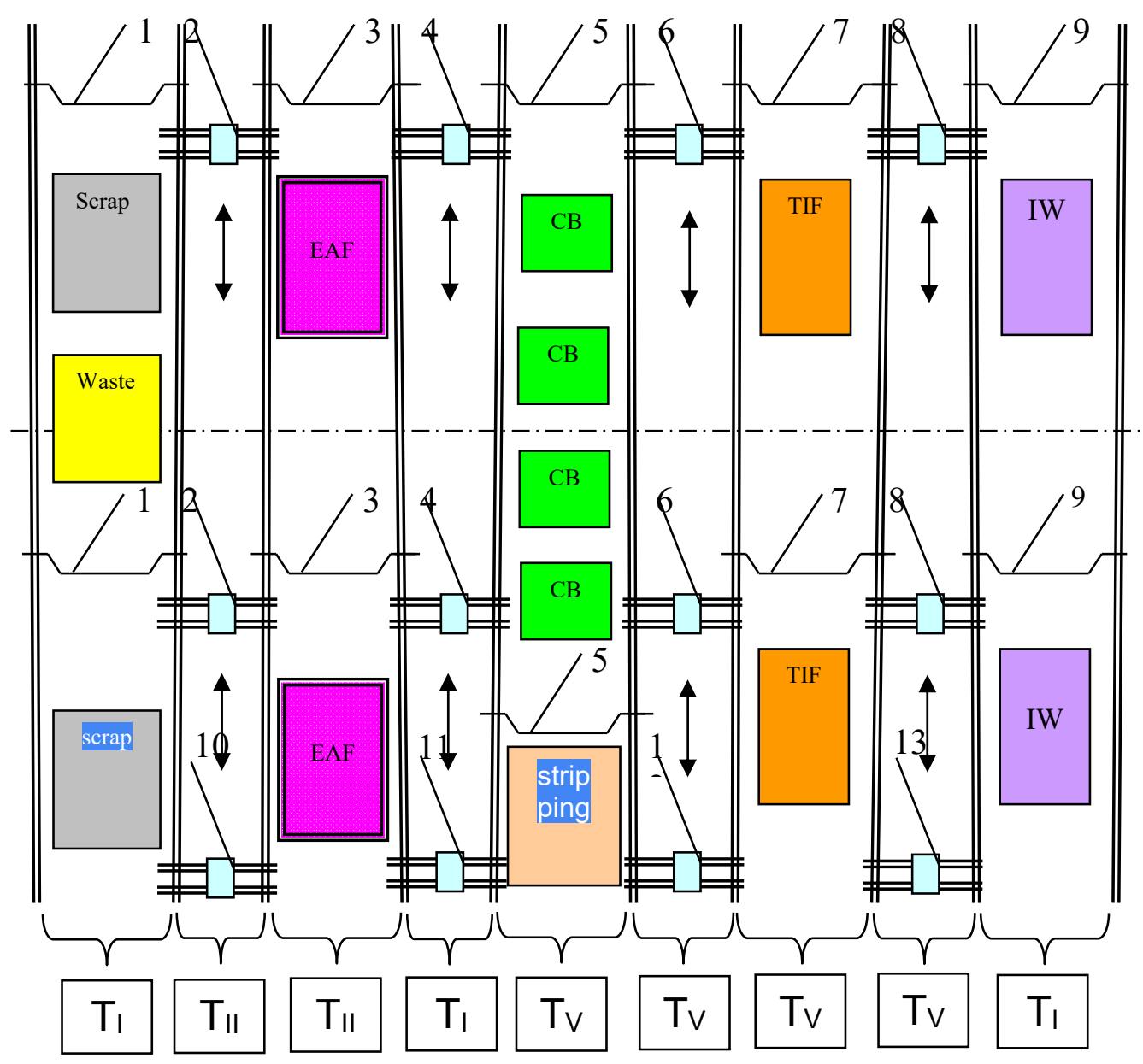

Fig. 3. Space organization in an electric steelworks.

The meaning of the notations is the following:

$T_{I}-$ Preparation / Dosing of metallic load (bay I).

$\mathrm{T}_{\text {II }}$ - Technical space for dosing-elaboration (bay II).

$\mathrm{T}_{\mathrm{III}}$ - Steel Elaboration (bay III).

$\mathrm{T}_{\mathrm{IV}}$ - Technical space for elaboration-casting (bay IV).

$\mathrm{T}_{\mathrm{V}}$ - Casting -stripping (bay V).

$\mathrm{T}_{\mathrm{VI}}$ - Technical space for casting- thermal treatment (bay VI).

$\mathrm{T}_{\mathrm{VII}}$ - Thermal treatment (bay VII).

$\mathrm{T}_{\mathrm{VIII}}$ - Technical space for thermal treatment-expedition (bay VIII).

$\mathrm{T}_{\mathrm{IX}}$ - Expedition (bay IX).

EAF -Electric Arc furnace for steel elaboration; CB - Casting Bridge; TTF - Thermal Treatment Furnace (group I and II); IW - Ingots Warehouse.

1 - Sliding bridges for Dosing-Load; 2, 10 - Transfers car for dosing-elaboration; 3 - Sliding bridges for Casting; 4, 11 - Transfer cars for elaboration-casting; 5 - Sliding bridges for Casting -stripping; 6, 12 - Transfer cars for casting-TC; 7 - Sliding bridges for TC; 8, 13 - Transfer cars for TC-expedition; 9 - Sliding bridges for expedition. 


\section{Conclusions}

An important role in making the enterprise more efficient in its relations with the external economic environment is the provision of a fluent and continuous supply and sales system. The spatial organization of the enterprise is of decisive importance for the fulfillment of this role.

The spatial organization of enterprises also has some disadvantages, of which we have to mention:

$>$ It requires high qualification of workers.

$>$ It requires a very high volume of transport and handling.

$>$ Quality technical control is much more complex in the conditions of a diversified production.

$>$ The production cycle of manufactured products is high.

In conclusion, the management of the spatial organization of enterprises in the metallic materials industry has to take into account both the optimal resolution of the internal (interior) organization of the enterprise (location and arrangement of sections, factories, workshops, aggregates, machinery etc.), and the external organization. This (the external organization) refers to the optimization of the enterprise's correlations with its external economic environment.

\section{References}

1. A. Ioana, M. Buzatu, A. Semenescu, Waste Management, 53, I-IV (2016)

2. A. Ioana, REVIEW OF RESEARCH AND SOCIAL INTERVENTION, 43, 169 2013)

3. A. Ioana, N. Constantin, P. Moldovan, IOP Conference Series: Materials Science and Engineering, 851 (2015)

4. M. Costoiu, A. Ioana, A. Semenescu, D. Marcu, Book Series: IOP Conference SeriesMaterials Science and Engineering, 161, 012104 (2016)

5. A. Ioana, A. Preda, A., L. Beşea, P. Moldovan, N. Constantin, U.P.B. Sci. Bull., Series $\mathrm{B}, \mathbf{7 8}, 3,185$ (2016) 